УДК 517.95

\author{
A. C. Cavalheiro
}

\title{
EXISTENCE AND UNIQUENESS OF THE SOLUTIONS FOR NONLINEAR DEGENERATE ELLIPTIC EQUATIONS IN WEIGHTED SOBOLEV SPACES
}

\begin{abstract}
A. C. Cavalheiro. Existence and uniqueness of solutions for degenerate nonlinear elliptic equations in weigthed Sobolev spaces, Mat. Stud. 45 (2016), 182-193.

In this paper we are interested in the existence and uniqueness of solutions for the Dirichlet
\end{abstract} problem associated to the degenerate nolinear elliptic equation

$$
-\sum_{j=1}^{n} D_{j}\left[\mathcal{A}_{j}(x, \nabla u) \omega_{2}(x)\right]+b(x, u) \omega_{1}(x)+g(x) u(x)=f_{0}(x)-\sum_{j=1}^{n} D_{j} f_{j}(x) \text { on } \Omega
$$

in the setting of the weighted Sobolev spaces $\mathrm{W}_{0}^{1, p}\left(\Omega, \omega_{1}, \omega_{2}\right)$.

1. Introduction. In this paper we prove the existence and uniqueness of (weak) solutions in the weighted Sobolev spaces $\mathrm{W}_{0}^{1, p}\left(\Omega, \omega_{1}, \omega_{2}\right)$ (see Definition 2) for the Dirichlet problem

$$
(P) \begin{cases}L u(x)=f_{0}(x)-\sum_{j=1}^{n} D_{j} f_{j}(x) & \text { in } \Omega, \\ u(x)=0 & \text { on } \partial \Omega,\end{cases}
$$

where $L$ is the partial differential operator

$$
L u(x)=-\sum_{j=1}^{n} D_{j}\left[\mathcal{A}_{j}(x, \nabla u(x)) \omega_{2}(x)\right]+b(x, u(x)) \omega_{1}(x)+g(x) u(x),
$$

$D_{j}=\partial / \partial x_{j}, \Omega$ is a bounded connected open set in $\mathbb{R}^{n}, \omega_{i}(i=1,2)$ are weights functions and the functions $\mathcal{A}=\left(\mathcal{A}_{1}, \ldots, \mathcal{A}_{n}\right): \Omega \times \mathbb{R}^{n} \rightarrow \mathbb{R}^{n}, b: \Omega \times \mathbb{R} \rightarrow \mathbb{R}, g: \Omega \rightarrow \mathbb{R}$ satisfy the following conditions:

(H1) $x \mapsto \mathcal{A}_{j}(x, \xi)$ is measurable on $\Omega$ for all $\xi \in \mathbb{R}^{n}, \xi \mapsto \mathcal{A}_{j}(x, \xi)$ is continuous on $\mathbb{R}^{n}$ for almost all $x \in \Omega$, and $\mathcal{A}_{j}(x, 0)=0$ for a.e. $x \in \Omega$;

(H2) there exist constants $p>2$ and $\lambda>0$ such that $\left[\mathcal{A}(x, \xi)-\mathcal{A}\left(x, \xi^{\prime}\right)\right] \cdot\left(\xi-\xi^{\prime}\right) \geq \lambda\left|\xi-\xi^{\prime}\right|^{p}$, whenever $\xi, \xi^{\prime} \in \mathbb{R}^{n}$, where $\mathcal{A}(x, \xi)=\left(\mathcal{A}_{1}(x, \xi), \ldots, \mathcal{A}_{n}(x, \xi)\right)$, a dot denote here the Euclidian scalar product in $\mathbb{R}^{n}$;

2010 Mathematics Subject Classification: 35J70, 35J60.

Keywords: nonlinear degenerate elliptic equation; weighted Sobolev space. doi:10.15330/ms.45.2.182-193

(C) A. C. Cavalheiro, 2016 
(H3) $|\mathcal{A}(x, \xi)| \leq K_{2}(x)+h_{2}(x)|\xi|^{p / p^{\prime}}$ such that $h_{2}$ and $K_{2}$ are nonnegative functions, with $h_{2} \in L^{\infty}(\Omega)$ and $K_{2} \in L^{p^{\prime}}\left(\Omega, \omega_{2}\right)\left(\right.$ with $\left.1 / p+1 / p^{\prime}=1\right) ;$

(H4) $x \mapsto b(x, \eta)$ is measurable on $\Omega$ for all $\eta \in \mathbb{R}, \eta \mapsto b(x, \eta)$ is continuous on $\mathbb{R}$ for almost all $x \in \Omega$, and $b(x, 0)=0$ for a.e. $x \in \Omega$;

(H5) there exists a constant $\Lambda>0$ such that $\left[b(x, \eta)-b\left(x, \eta^{\prime}\right)\right]\left(\eta-\eta^{\prime}\right) \geq \Lambda\left|\eta-\eta^{\prime}\right|^{p}$, whenever $\eta, \eta^{\prime} \in \mathbb{R}$

(H6) $|b(x, \eta)| \leq K_{1}(x)+h_{1}(x)|\eta|^{p / p^{\prime}}$, where $K_{1}$ and $h_{1}$ are nonnegative functions such that $K_{1} \in L^{p^{\prime}}\left(\Omega, \omega_{1}\right)$ and $h_{1} \in L^{\infty}(\Omega)$;

(H7) $g / \omega_{1} \in L^{q}\left(\Omega, \omega_{1}\right)$, where $1 / q=1 / p^{\prime}-1 / p$, and $g(x) \geq 0$ a.e. $x \in \Omega$;

(H8) $\omega_{1}$ and $\omega_{2}$ are weights from the Muckenhoupt class $A_{p}$, with $2<p<\infty$;

(H9) $f_{0} / \omega_{1} \in L^{p^{\prime}}\left(\Omega, \omega_{1}\right)$ and $f_{j} / \omega_{2} \in L^{p^{\prime}}\left(\Omega, \omega_{2}\right)(j=1, \ldots, n)$.

Under a weight, we mean a locally integrable function $\omega$ on $\mathbb{R}^{n}$ such that $0<\omega(x)<\infty$ for a.e. $x \in \mathbb{R}^{n}$ (see [13]). Every weight $\omega$ gives rise to a measure on the measurable subsets on $\mathbb{R}^{n}$ through integration. This measure will be denoted by $\mu$. Thus, $\mu(E)=\int_{E} \omega(x) d x$ for all measurable sets $E \subset \mathbb{R}^{n}$.

In general, the Sobolev spaces $\mathrm{W}^{k, p}(\Omega)$ without weights occur as spaces of solutions for elliptic and parabolic partial differential equations. For the degenerate partial differential equations, i.e., the equations with various types of singularities in coefficients, it is natural to look for solutions in the weighted Sobolev spaces (see [3], [5] and [6]).

A class of weights, which is particulary well understood, is the class of $A_{p}$-weights (or the Muckenhoupt class) these classes weve introduced by B. Muckenhoupt (see [11]). They have found many usefull applications in harmonic analysis (see [12]). Another reason for studying $A_{p}$-weights is the fact that powers of distance to submanifolds of $\mathbb{R}^{n}$ often belong to $A_{p}$ (see [9]). There are, in fact, many interesting examples of weights (see [8] for $p$-admissible weights).

In the non-degenerate case (i.e., with $\left.\omega_{1}(x)=\omega_{2}(x)=1\right)$ the problem $(\mathrm{P})$ has been studied in [1] and [2].

Remark 1. (a) From (H2) and the fact that $\mathcal{A}_{j}(x, 0)=0$ for a.e. $x \in \Omega$, we have

$$
\mathcal{A}(x, \xi) \cdot \xi=(\mathcal{A}(x, \xi)-\mathcal{A}(x, 0)) \cdot(\xi-0) \geq \lambda|\xi|^{p}
$$

(b) Since $b(x, 0)=0$ for a.e. $x \in \Omega$, we deduce from (H5) that

$$
b(x, \eta) \eta=(b(x, \eta)-b(x, 0))(\eta-0) \geq \Lambda|\eta|^{p} .
$$

Definition 1. We say that an element $u \in W_{0}^{1, p}\left(\Omega, \omega_{1}, \omega_{2}\right)$ is a (weak) solution of problem $(P)$ if

$$
\begin{aligned}
& \sum_{j=1}^{n} \int_{\Omega} \mathcal{A}_{j}(x, \nabla u(x)) D_{j} \varphi(x) \omega_{2}(x) d x+\int_{\Omega} b(x, u(x)) \varphi(x) \omega_{1}(x) d x+ \\
& +\int_{\Omega} g(x) u(x) \varphi(x) d x=\int_{\Omega} f_{0}(x) \varphi(x) d x+\sum_{j=1}^{n} \int_{\Omega} f_{j}(x) D_{j} \varphi(x) d x,
\end{aligned}
$$

for all $\varphi \in W_{0}^{1, p}\left(\Omega, \omega_{1}, \omega_{2}\right)$. 
The following theorem will be proved in Section 3 .

Theorem 1. Suppose that conditions (H1)-(H9) hold. Then the problem (P) has the unique solution $u \in W_{0}^{1, p}\left(\Omega, \omega_{1}, \omega_{2}\right)$. Moreover,

$$
\|u\|_{W_{0}^{1, p}\left(\Omega, \omega_{1}, \omega_{2}\right)} \leq \frac{1}{\gamma^{p^{\prime} / p}}\left(\left\|\frac{f_{0}}{\omega_{1}}\right\|_{L^{p^{\prime}\left(\Omega, \omega_{1}\right)}}+\sum_{j=1}^{n}\left\|\frac{f_{j}}{\omega_{2}}\right\|_{L^{p^{\prime}\left(\Omega, \omega_{2}\right)}}\right)^{p^{\prime} / p}
$$

where $\gamma=\min \{\lambda, \Lambda\}$.

Corollary 1. Under the assumptions of Theorem 1, if $u_{1} \in W_{0}^{1, p}\left(\Omega, \omega_{1}, \omega_{2}\right)$ is a solution of the problem

$$
\left(P_{1}\right) \begin{cases}L u_{1}(x)=f_{0}(x)-\sum_{j=1}^{n} D_{j} f_{j}(x) & \text { in } \Omega, \\ u_{1}(x)=0 & \text { on } \partial \Omega\end{cases}
$$

$u_{2} \in W_{0}^{1, p}\left(\Omega, \omega_{1}, \omega_{2}\right)$ is a solutions of

$$
\left(P_{2}\right) \begin{cases}L u_{2}(x)=\tilde{f}_{0}(x)-\sum_{j=1}^{n} D_{j} \tilde{f}_{j}(x) & \text { in } \Omega \\ u_{2}(x)=0 & \text { on } \partial \Omega\end{cases}
$$

and $\gamma=\min \{\lambda, \Lambda\}$, then

$$
\left\|u_{1}-u_{2}\right\|_{W_{0}^{1, p}\left(\Omega, \omega_{1}, \omega_{2}\right)} \leq \frac{1}{\gamma^{p / p^{\prime}}}\left(\left\|\frac{f_{0}-\tilde{f}_{0}}{\omega_{1}}\right\|_{L^{p^{\prime}\left(\Omega, \omega_{1}\right)}}+\sum_{j=1}^{n}\left\|\frac{f_{j}-\tilde{f}_{j}}{\omega_{2}}\right\|_{L^{p^{\prime}}\left(\Omega, \omega_{2}\right)}\right)^{p / p^{\prime}} .
$$

Corollary 2. Let the assumptions of Theorem 1 be fulfilled, and let $\left\{f_{0 m}\right\}$ and $\left\{f_{j m}\right\}$ $(j=1, \ldots, n)$ be sequences of functions satisfying $\frac{f_{0 m}}{\omega_{1}} \rightarrow \frac{f_{0}}{\omega_{1}}$ in $L^{p^{\prime}}\left(\Omega, \omega_{1}\right)$ and $\frac{f_{j m}}{\omega_{2}} \rightarrow \frac{f_{j}}{\omega_{2}}$ in $L^{p^{\prime}}\left(\Omega, \omega_{2}\right)$. If $u_{m} \in W_{0}^{1, p}\left(\Omega, \omega_{1}, \omega_{2}\right)$ is a solution of the problem

$$
\left(P_{m}\right) \begin{cases}L u_{m}(x)=f_{0 m}(x)-\sum_{j=1}^{n} D_{j} f_{j m}(x) & \text { in } \Omega, \\ u_{m}(x)=0 & \text { on } \partial \Omega,\end{cases}
$$

then $u_{m} \rightarrow u$ in $W_{0}^{1, p}\left(\Omega, \omega_{1}, \omega_{2}\right)$, where $u$ is a solution of $(P)$.

2. Definitions and basic results. Let $\omega$ be a locally integrable nonnegative function in $\mathbb{R}^{n}$ and assume that $0<\omega<\infty$ almost everywhere. We say that $\omega$ belongs to the Muckenhoupt

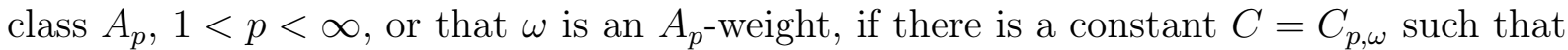

$$
\left(\frac{1}{|B|} \int_{B} \omega(x) d x\right)\left(\frac{1}{|B|} \int_{B} \omega^{1 /(1-p)}(x) d x\right)^{p-1} \leq C
$$

for all balls $B \subset \mathbb{R}^{n}$, where $|$.$| denotes the n$-dimensional Lebesgue measure in $\mathbb{R}^{n}$. If $1<$ $q \leq p$, then $A_{q} \subset A_{p}$ (see [7], [8] or [12] for more information about $A_{p}$-weights).

As an example of $A_{p}$-weight, the function $\omega(x)=|x|^{\alpha}, x \in \mathbb{R}^{n}$, is in $A_{p}$ if and only if $-n<\alpha<n(p-1)$ (see Corollary 4.4, Chapter IX in [12]).

The measure $\mu$ and the Lebesgue measure $|\cdot|$ are mutually absolutely continuous, i.e., they have the same zero sets $(\mu(E)=0$ if and only if $|E|=0)$; so there is no need to specify the measure when using the ubiquitous expression almost everywhere and almost every, both abbreviated a.e.. 
Definition 2. Let $\omega$ be a weight, and let $\Omega \subset \mathbb{R}^{n}$ be open. For $1<p<\infty$ we define $L^{p}(\Omega, \omega)$ as the set of measurable functions $f$ on $\Omega$ such that

$$
\|f\|_{L^{p}(\Omega, \omega)}=\left(\int_{\Omega}|f(x)|^{p} \omega(x) d x\right)^{1 / p}<\infty .
$$

If $\omega \in A_{p}, 1<p<\infty$, then $\omega^{-1 /(p-1)}$ is locally integrable and $L^{p}(\Omega, \omega) \subset L_{\text {loc }}^{1}(\Omega)$ for every open set $\Omega$ (see Remark 1.2.4 in [13]). It thus makes sense to talk about weak derivatives of functions in $L^{p}(\Omega, \omega)$.

Definition 3. Let $\Omega \subset \mathbb{R}^{n}$ be a bounded open set, and let $\omega_{1}$ and $\omega_{2}$ be $A_{p}$-weights $(1<p<$ $\infty)$. We define the weighted Sobolev space $W^{1, p}\left(\Omega, \omega_{1}, \omega_{2}\right)$ as the set of functions $u \in L^{p}\left(\Omega, \omega_{1}\right)$ with the weak derivatives $D_{j} u \in L^{p}\left(\Omega, \omega_{2}\right)(j=1, \ldots, n)$. The norm of $u$ in $W^{1, p}\left(\Omega, \omega_{1}, \omega_{2}\right)$ is defined by

$$
\|u\|_{W^{1, p}\left(\Omega, \omega_{1}, \omega_{2}\right)}=\left(\int_{\Omega}|u(x)|^{p} \omega_{1}(x) d x+\int_{\Omega}|\nabla u(x)|^{p} \omega_{2}(x) d x\right)^{1 / p} .
$$

The space $W_{0}^{1, p}\left(\Omega, \omega_{1}, \omega_{2}\right)$ is the clousure of $C_{0}^{\infty}(\Omega)$ with respect norm (1). Equipped with this norm, $W_{0}^{1, p}\left(\Omega, \omega_{1}, \omega_{2}\right)$ is a reflevixe Banach space (see [10] for more information about the spaces $\left.W^{1, p}\left(\Omega, \omega_{1}, \omega_{2}\right)\right)$. The dual space of $W_{0}^{1, p}\left(\Omega, \Omega_{1}, \omega_{2}\right)$ is the space

$$
\begin{gathered}
{\left[W_{0}^{1, p}\left(\Omega, \omega_{2}, \omega_{2}\right)\right]^{*}=} \\
=\left\{T=f_{0}-\operatorname{div}(F), F=\left(f_{1}, \ldots, f_{n}\right): \frac{f_{0}}{\omega_{1}} \in L^{p^{\prime}}\left(\Omega, \omega_{1}\right), \frac{f_{j}}{\omega_{2}} \in L^{p^{\prime}}\left(\Omega, \omega_{2}\right), j=1, \ldots, n\right\} .
\end{gathered}
$$

In this paper we use the following theorem.

Theorem 2. Let $\omega \in A_{p}, 1<p<\infty$, and let $\Omega$ be a bounded open set in $\mathbb{R}^{n}$. If $u_{m} \rightarrow u$ in $L^{p}(\Omega, \omega)$, then there exist a subsequence $\left\{u_{m_{k}}\right\}$ and a function $\Phi \in L^{p}(\Omega, \omega)$ such that

(i) $u_{m_{k}}(x) \rightarrow u(x), m_{k} \rightarrow \infty$, a.e. on $\Omega$;

(ii) $\left|u_{m_{k}}(x)\right| \leq \Phi(x)$ a.e. on $\Omega$.

The proof of this theorem follows the lines of Theorem 2.8.1 in [4].

Proof of Theorem 1. The basic idea is to reduce the problem (P) to an operator equation $A u=T$ and apply the theorem below.

Theorem 3. Let $A: X \rightarrow X^{*}$ be a monotone, coercive and hemicontinuous operator on the real, separable, reflexive Banach space $X$. Then the following assertions hold:

(a) for each $T \in X^{*}$ the equation $A u=T$ has a solution $u \in X$;

(b) if the operator $A$ is strictly monotone, then equation $A u=T$ is uniquely solvable in $X$ (Theorem 26.A in [15]).

To prove Theorem 1 , we define $B, B_{1}, B_{2}: W_{0}^{1, p}\left(\Omega, \omega_{1}, \omega_{2}\right) \times W_{0}^{1, p}\left(\Omega, \omega_{1}, \omega_{2}\right) \rightarrow \mathbb{R}$ and $T: W_{0}^{1, p}\left(\Omega, \omega_{1}, \omega_{2}\right) \rightarrow \mathbb{R}$ by

$$
B(u, \varphi)=B_{1}(u, \varphi)+B_{2}(u, \varphi)
$$




$$
\begin{gathered}
B_{2}(u, \varphi)=\sum_{j=1}^{n} \int_{\Omega} \mathcal{A}_{j}(x, \nabla u) D_{j} \varphi \omega_{2} d x=\int_{\Omega} \mathcal{A}(x, \nabla u) . \nabla \varphi \omega_{2} d x, \\
B_{1}(u, \varphi)=\int_{\Omega} b(x, u(x)) \varphi(x) \omega_{1}(x) d x+\int_{\Omega} g(x) \varphi(x) u(x) d x, \\
T(\varphi)=\int_{\Omega} f_{0}(x) \varphi(x) d x+\sum_{j=1}^{n} \int_{\Omega} f_{j}(x) D_{j} \varphi(x) d x .
\end{gathered}
$$

Then $u \in W_{0}^{1, p}\left(\Omega, \omega_{1}, \omega_{2}\right)$ is a (weak) solution of problem $(\mathrm{P})$ if and only if

$$
B(u, \varphi)=T(\varphi) \text { for all } \varphi \in W_{0}^{1, p}\left(\Omega, \omega_{1}, \omega_{2}\right) .
$$

Step 1. For $j=1, \ldots, n$ we define the operator $F_{j}: W_{0}^{1, p}\left(\Omega, \omega_{1}, \omega_{2}\right) \rightarrow L^{p^{\prime}}\left(\Omega, \omega_{2}\right)$ as $\left(F_{j} u\right)(x)=\mathcal{A}_{j}(x, \nabla u(x))$. We now show that the operator $F_{j}$ is bounded and continuous.

(i) From (H3) we obtain

$$
\begin{gathered}
\left\|F_{j} u\right\|_{L^{p^{\prime}\left(\Omega, \omega_{2}\right)}}^{p^{\prime}}=\int_{\Omega}\left|F_{j} u(x)\right|^{p^{\prime}} \omega_{2} d x=\int_{\Omega}\left|\mathcal{A}_{j}(x, \nabla u)\right|^{p^{\prime}} \omega_{2} d x \leq \int_{\Omega}\left(K_{2}+h_{2}|\nabla u|^{p / p^{\prime}}\right)^{p^{\prime}} \omega_{2} d x \leq \\
\leq C_{p} \int_{\Omega}\left(K_{2}^{p^{\prime}}+h_{2}^{p^{\prime}}|\nabla u|^{p}\right) \omega_{2} d x=C_{p}\left[\int_{\Omega} K_{2}^{p^{\prime}} \omega_{2} d x+\int_{\Omega} h_{2}^{p^{\prime}}|\nabla u|^{p} \omega_{2} d x\right] \leq \\
\leq C_{p}\left[\left\|K_{2}\right\|_{L^{p^{\prime}\left(\Omega, \omega_{2}\right)}}^{p^{\prime}}+\left\|h_{2}\right\|_{L^{\infty}(\Omega)}^{p^{\prime}}\|u\|_{W_{0}^{1, p}\left(\Omega, \omega_{1}, \omega_{2}\right)}^{p}\right]
\end{gathered}
$$

where the constant $C_{p}$ depends on $p$ only. Therefore,

$$
\left\|F_{j} u\right\|_{L^{p^{\prime}\left(\Omega, \omega_{2}\right)}} \leq C_{p}\left(\left\|K_{2}\right\|_{L^{p^{\prime}\left(\Omega, \omega_{2}\right)}}+\left\|h_{2}\right\|_{L^{\infty}(\Omega)}\|u\|_{W_{0}^{1, p}\left(\Omega, \omega_{1}, \omega_{2}\right)}^{p / p^{\prime}}\right) .
$$

(ii) Let $u_{m} \rightarrow u$ in $W_{0}^{1, p}\left(\Omega, \omega_{1}, \omega_{2}\right)$ as $m \rightarrow \infty$. We need to show that $F_{j} u_{m} \rightarrow F_{j} u$ in $L^{p^{\prime}}\left(\Omega, \omega_{2}\right)$. We will apply the Lebesgue Dominated Theorem. If $u_{m} \rightarrow u$ in $W_{0}^{1, p}\left(\Omega, \omega_{1}, \omega_{2}\right)$, then $u_{m} \rightarrow u$ in $L^{p}\left(\Omega, \omega_{1}\right)$ and $\left|\nabla u_{m}\right| \rightarrow|\nabla u|$ in $L^{p}\left(\Omega, \omega_{2}\right)$. By Theorem 2 , there exist a subsequence $\left\{u_{m_{k}}\right\}$, functions $\Phi_{1} \in L^{p}\left(\Omega, \omega_{1}\right)$ and $\Phi_{2} \in L^{p}\left(\Omega, \omega_{2}\right)$ such that

$$
\begin{gathered}
u_{m_{k}}(x) \rightarrow u(x) \text { a.e. in } \Omega,\left|u_{m_{k}}(x)\right| \leq \Phi_{1}(x) \text { a.e. in } \Omega, \\
D_{j} u_{m_{k}}(x) \rightarrow D_{j} u(x) \text { a.e. in } \Omega(j=1, \ldots, n), \quad\left|\nabla u_{m_{k}}(x)\right| \leq \Phi_{2}(x) \text { a.e. in } \Omega .
\end{gathered}
$$

Next, applying (H3) yeilds

$$
\begin{gathered}
\left|F_{j} u_{m_{k}}-F_{j} u\right|^{p^{\prime}} \omega_{2}=\left|\mathcal{A}_{j}\left(x, \nabla u_{m_{k}}\right)-\mathcal{A}_{j}(x, \nabla u)\right|^{p^{\prime}} \omega_{2} \leq \\
\leq C_{p}\left(\left|\mathcal{A}_{j}\left(x, \nabla u_{m_{k}}\right)\right|^{p^{\prime}}+\left|\mathcal{A}_{j}(x, \nabla u)\right|^{p^{\prime}}\right) \omega_{2} \leq \\
\leq C_{p}\left[\left(K_{2}+h_{2}\left|\nabla u_{m_{k}}\right|^{p / p^{\prime}}\right)^{p^{\prime}}+\left(K_{2}+h_{2}|\nabla u|^{p / p^{\prime}}\right)^{p^{\prime}}\right] \omega_{2} \leq \\
\leq C_{p}^{2}\left[K_{2}^{p^{\prime}}+\left\|h_{2}\right\|_{L^{\infty}(\Omega)}^{p^{\prime}}\left|\nabla u_{m_{k}}\right|^{p}+K_{2}^{p^{\prime}}+\left\|h_{2}\right\|_{L^{\infty}(\Omega)}^{p^{\prime}}|\nabla u|^{p}\right] \omega_{2} \leq \\
\leq 2 C_{p}^{2}\left[K_{2}^{p^{\prime}}+\left\|h_{2}\right\|_{L^{\infty}(\Omega)}^{p^{\prime}}\left|\Phi_{2}\right|^{p}\right] \omega_{2} \in L^{1}(\Omega) .
\end{gathered}
$$


By condition (H1), we have

$$
F_{j} u_{m_{k}}(x)=\mathcal{A}_{j}\left(x, \nabla u_{m_{k}}(x)\right) \rightarrow \mathcal{A}_{j}(x, \nabla u(x))=F_{j} u(x),
$$

as $m_{k} \rightarrow+\infty$. Therefore, by the Lebesgue Dominated Convergence Theorem, we obtain $\left\|F_{j} u_{m_{k}}-F_{j} u\right\|_{L^{p^{\prime}\left(\Omega, \omega_{2}\right)}} \rightarrow 0$, that is, $F_{j} u_{m_{k}} \rightarrow F_{j} u$ in $L^{p^{\prime}}\left(\Omega, \omega_{2}\right)$. We conclude from the Convergence Principle in Banach spaces (see Proposition 10.13 in [14]) that

$$
F_{j} u_{m} \rightarrow F_{j} u \text { in } L^{p^{\prime}}\left(\Omega, \omega_{2}\right) .
$$

Step 2. We define the operator $G: W_{0}^{1, p}\left(\Omega, \omega_{1}, \omega_{2}\right) \rightarrow L^{p^{\prime}}\left(\Omega, \omega_{1}\right)$ by $(G u)(x)=b(x, u(x))$.

This operator is continuous and bounded. In fact,

(i) By (H6), we obtain

$$
\begin{gathered}
\|G u\|_{L^{p^{\prime}\left(\Omega, \omega_{1}\right)}}^{p^{\prime}}=\int_{\Omega}|G u|^{p^{\prime}} \omega_{1} d x=\int_{\Omega}|b(x, u)|^{p^{\prime}} \omega_{1} d x \leq \int_{\Omega}\left(K_{1}+h_{1}|u|^{p / p^{\prime}}\right)^{p^{\prime}} \omega_{1} d x \leq \\
\leq C_{p} \int_{\Omega}\left(K_{1}^{p^{\prime}}+h_{1}^{p^{\prime}}|u|^{p}\right) \omega_{1} d x=C_{p}\left[\int_{\Omega} K_{1}^{p^{\prime}} \omega_{1} d x+\int_{\Omega} h_{1}^{p^{\prime}}|u|^{p} \omega_{1} d x\right] \leq \\
\leq C_{p}\left(\left\|K_{1}\right\|_{L^{p^{\prime}\left(\Omega, \omega_{1}\right)}}^{p^{\prime}}+\left\|h_{2}\right\|_{L^{\infty}(\Omega)}^{p^{\prime}}\|u\|_{W_{0}^{1, p}\left(\Omega, \omega_{1}, \omega_{2}\right)}^{p}\right) .
\end{gathered}
$$

(ii) Applying (H6) together with a similar argument as in Step 1(ii) yields

$$
\begin{gathered}
\left|G u_{m_{k}}-G u\right|^{p^{\prime}} \omega_{1}=\left|b\left(x, u_{m_{k}}\right)-b(x, u)\right|^{p^{\prime}} \omega_{1} \leq C_{p}\left(\left|b\left(x, u_{m_{k}}\right)\right|^{p^{\prime}}+|b(x, u)|^{p^{\prime}}\right) \omega_{1} \leq \\
\leq C_{p}\left[\left(K_{1}+h_{1}\left|u_{m_{k}}\right|^{p / p^{\prime}}\right)^{p^{\prime}}+\left(K_{1}+h_{1}|u|^{p / p^{\prime}}\right)^{p^{\prime}}\right] \omega_{1} \leq \\
\leq C_{p}^{2}\left[K_{1}^{p^{\prime}}+\left\|h_{1}\right\|_{L^{\infty}(\Omega)}^{p^{\prime}}\left|u_{m_{k}}\right|^{p}+K_{1}^{p^{\prime}}+\left\|h_{1}\right\|_{L^{\infty}(\Omega)}^{p^{\prime}}|u|^{p}\right] \omega_{1} \leq \\
\leq 2 C_{p}^{2}\left[K_{1}^{p^{\prime}}+\left\|h_{1}\right\|_{L^{\infty}(\Omega)}^{p^{\prime}}\left|\Phi_{1}\right|^{p}\right] \omega_{1} \in L^{1}(\Omega) .
\end{gathered}
$$

Repeated application of the Convergence Principle in Banach Spaces given as: if $u_{m} \rightarrow u$ in $W_{0}^{1, p}\left(\Omega, \omega_{1}, \omega_{2}\right)$ we obtain

$$
G u_{m} \rightarrow G u \text { in } L^{p^{\prime}}\left(\Omega, \omega_{1}\right) .
$$

Step 3. By (H9) we have

$$
\begin{gathered}
|T(\varphi)| \leq \int_{\Omega}\left|f_{0}\right||\varphi| d x+\sum_{j=1}^{n} \int_{\Omega}\left|f_{j}\right|\left|D_{j} \varphi\right| d x=\int_{\Omega} \frac{\left|f_{0}\right|}{\omega_{1}}|\varphi| \omega_{1} d x+\sum_{j=1}^{n} \int_{\Omega} \frac{\left|f_{j}\right|}{\omega_{2}}\left|D_{j} \varphi\right| \omega_{2} d x \leq \\
\leq\left\|f_{0} / \omega_{1}\right\|_{L^{p^{\prime}\left(\Omega, \omega_{1}\right)}}\|\varphi\|_{L^{p}\left(\Omega, \omega_{1}\right)}+\sum_{j=1}^{n}\left\|f_{j} / \omega_{2}\right\|_{L^{p^{\prime}\left(\Omega, \omega_{2}\right)}}\left\|D_{j} \varphi\right\|_{L^{p}\left(\Omega, \omega_{2}\right)} \leq \\
\leq\left(\left\|f_{0} / \omega_{1}\right\|_{L^{p^{\prime}}\left(\Omega, \omega_{1}\right)}+\sum_{j=1}^{n}\left\|f_{j} / \omega_{2}\right\|_{L^{p^{\prime}}\left(\Omega, \omega_{2}\right)}\right)\|\varphi\|_{W_{0}^{1, p}\left(\Omega, \omega_{1}, \omega_{2}\right)} .
\end{gathered}
$$


Moreover, using (H3), (H6), (H7) and the generalized Hölder inequality, we also have

$$
\begin{gathered}
|B(u, \varphi)| \leq\left|B_{1}(u, \varphi)\right|+\left|B_{2}(u, \varphi)\right| \leq \\
\leq \sum_{j=1}^{n} \int_{\Omega}\left|\mathcal{A}_{j}(x, \nabla u)\right|\left|D_{j} \varphi\right| \omega_{2} d x+\int_{\Omega}|b(x, u)||\varphi| \omega_{1} d x+\int_{\Omega}|g||u||\varphi| d x .
\end{gathered}
$$

The right hand side of (5) admits the estimation:

$$
\begin{gathered}
\int_{\Omega}|\mathcal{A}(x, \nabla u) \| \nabla \varphi| \omega_{2} d x \leq \int_{\Omega}\left(K_{2}+h_{2}|\nabla u|^{p / p^{\prime}}\right)|\nabla \varphi| \omega_{2} d x \leq \\
\leq\left\|K_{2}\right\|_{L^{p^{\prime}\left(\Omega, \omega_{2}\right)}}\|\nabla \varphi\|_{L^{p}\left(\Omega, \omega_{2}\right)}+\left\|h_{2}\right\|_{L^{\infty}(\Omega)}\|\nabla u\|_{L^{p}\left(\Omega, \omega_{2}\right)}^{p / p^{\prime}}\|\nabla \varphi\|_{L^{p}\left(\Omega, \omega_{2}\right)} \leq \\
\leq\left(\left\|K_{2}\right\|_{L^{p^{\prime}\left(\Omega, \omega_{2}\right)}}+\left\|h_{2}\right\|_{L^{\infty}(\Omega)}\|u\|_{W_{0}^{1, p}\left(\Omega, \omega_{1}, \omega_{2}\right)}^{p / p^{\prime}}\right)\|\varphi\|_{W_{0}^{1, p}\left(\Omega, \omega_{1}, \omega_{2}\right)}, \\
\quad \int_{\Omega}|b(x, u) \| \varphi| \omega_{1} d x \leq \int_{\Omega}\left(K_{1}+h_{1}|u|^{p / p^{\prime}}\right)|\varphi| y \omega_{1} y d x \leq \\
\leq \int_{\Omega} K_{1} y|\varphi| y \omega_{1} y d x+\left\|h_{1}\right\|_{L^{\infty}(\Omega)} \int_{\Omega}|u|^{p / p y^{\prime}}|\varphi| y \omega_{1} y d x \leq \\
\leq\left(\left\|K_{1}\right\|_{L^{p^{\prime}\left(\Omega, \omega_{1}\right)}}+\left\|h_{1}\right\|_{L^{\infty}(\Omega)}\|u\|_{W_{0}^{1, p}\left(\Omega, \omega_{1}, \omega_{2}\right)}^{p / p^{\prime}}\right)\|\varphi\|_{W_{0}^{1, p}\left(\Omega, \omega_{1}, \omega_{2}\right)}
\end{gathered}
$$

and, by (H2), since $1 / q+1 / p+1 / p^{\prime}=1$ (by (H7)),

$$
\begin{aligned}
\int_{\Omega}|g||u||\varphi| d x & =\int_{\Omega} \frac{|g|}{\omega_{1}}\left|u\left\|\varphi \mid \omega_{1} d x \leq\right\| g / \omega_{1}\left\|_{L^{q}\left(\Omega, \omega_{1}\right)}\right\| u\left\|_{L^{p}\left(\Omega, \omega_{1}\right)}\right\| \varphi \|_{L^{p}\left(\Omega, \omega_{1}\right)} \leq\right. \\
& \leq\left\|g / \omega_{1}\right\|_{L^{q}\left(\Omega, \omega_{1}\right)}\|u\|_{W_{0}^{1, p}\left(\Omega, \omega_{1}, \omega_{2}\right)}\|\varphi\|_{W_{0}^{1, p}\left(\Omega, \omega_{1}, \omega_{2}\right)} .
\end{aligned}
$$

Finally, we obtain

$$
\begin{aligned}
|B(u, \varphi)| \leq & {\left[\left\|K_{1}\right\|_{L^{p^{\prime}\left(\Omega, \omega_{1}\right)}}+\left\|h_{1}\right\|_{L^{\infty}(\Omega)}\|u\|_{W_{0}^{1, p}\left(\Omega, \omega_{1}, \omega_{2}\right)}^{p / p^{\prime}}+\left\|h_{2}\right\|_{L^{\infty}(\Omega)}\|u\|_{W_{0}^{1, p}\left(\Omega, \omega_{1}, \omega_{2}\right)}^{p / p^{\prime}}+\right.} \\
& \left.+\left\|K_{2}\right\|_{L^{p^{\prime}\left(\Omega, \omega_{2}\right)}}+\left\|g / \omega_{1}\right\|_{L^{q}\left(\Omega, \omega_{1}\right)}\|u\|_{W_{0}^{1, p}\left(\Omega, \omega_{1}, \omega_{2}\right)}\right]\|\varphi\|_{W_{0}^{1, p}\left(\Omega, \omega_{1}, \omega_{2}\right)}
\end{aligned}
$$

for all $u, \varphi \in W_{0}^{1, p}\left(\Omega, \omega_{1}, \omega_{2}\right)$.

Since $B(u,$.$) is linear, for each u \in W_{0}^{1, p}\left(\Omega, \omega_{1}, \omega_{2}\right)$, there exists a linear continuous functional on $W_{0}^{1, p}\left(\Omega, \omega_{1}, \omega_{2}\right)$ denoted by $A u$ such that $\langle A u, \varphi\rangle=B(u, \varphi)$, for all $\varphi \in$ $W_{0}^{1, p}\left(\Omega, \omega_{1}, \omega_{2}\right)$. Here $\langle f, x\rangle$ denotes the value of the linear functional $f$ at the point $x$. Moreover

$$
\begin{gathered}
\|A u\|_{*} \leq\left\|K_{1}\right\|_{L^{p^{\prime}\left(\Omega, \omega_{1}\right)}}+\left\|K_{2}\right\|_{L^{p^{\prime}\left(\Omega, \omega_{2}\right)}}+\left\|h_{1}\right\|_{L^{\infty}(\Omega)}\|u\|_{W_{0}^{1, p}\left(\Omega, \omega_{1}, \omega_{2}\right)}^{p / p^{\prime}}+ \\
+\left\|h_{2}\right\|_{L^{\infty}(\Omega)}\|u\|_{W_{0}^{1, p}\left(\Omega, \omega_{1}, \omega_{2}\right)}^{p / p^{\prime}}+\left\|g / \omega_{1}\right\|_{L^{q}\left(\Omega, \omega_{1}\right)}\|u\|_{W_{0}^{1, p}\left(\Omega, \omega_{1}, \omega_{2}\right)} .
\end{gathered}
$$

Hence, we obtain the operator

$$
A: W_{0}^{1, p}\left(\Omega, \omega_{1}, \omega_{2}\right) \rightarrow\left[W_{0}^{1, p}\left(\Omega, \omega_{1}, \omega_{2}\right)\right]^{*}, \quad u \mapsto A u .
$$


Consequently, problem $(\mathrm{P})$ is equivalent to the operator equation

$$
u \in W_{0}^{1, p}\left(\Omega, \omega_{1}, \omega_{2}\right): A u=T .
$$

Step 4. Using condition (H2), (H5) and (H7), we have

$$
\begin{gathered}
\left\langle A u_{1}-A u_{2}, u_{1}-u_{2}\right\rangle=B\left(u_{1}, u_{1}-u_{2}\right)-B\left(u_{2}, u_{1}-u_{2}\right)= \\
=\int_{\Omega} \mathcal{A}\left(x, \nabla u_{1}\right) \cdot \nabla\left(u_{1}-u_{2}\right) \omega_{2} d x+\int_{\Omega} b\left(x, u_{1}\right)\left(u_{1}-u_{2}\right) \omega_{1} d x+\int_{\Omega}\left(u_{1}-u_{2}\right) g u_{1} d x- \\
-\int_{\Omega} \mathcal{A}\left(x, \nabla u_{2}\right) \cdot \nabla\left(u_{1}-u_{2}\right) \omega_{2} d x-\int_{\Omega} b\left(x, u_{2}\right)\left(u_{1}-u_{2}\right) \omega_{1} d x-\int_{\Omega} g\left(u_{1}-u_{2}\right) u_{2} d x \\
=\int_{\Omega}\left(\mathcal{A}\left(x, \nabla u_{1}\right)-\mathcal{A}\left(x, \nabla u_{2}\right)\right) \cdot \nabla\left(u_{1}-u_{2}\right) \omega_{2} d x+ \\
+\int_{\Omega}\left(b\left(x, u_{1}\right)-b\left(x, u_{2}\right)\right)\left(u_{1}-u_{2}\right) \omega_{1} d x+\int_{\Omega} g\left(u_{1}-u_{2}\right)^{2} d x \geq \\
\geq \lambda \int_{\Omega}\left|\nabla\left(u_{1}-u_{2}\right)\right|^{p} \omega_{2} d x+\Lambda \int_{\Omega}\left|u_{1}-u_{2}\right|^{p} \omega_{1} d x \geq \gamma\left\|u_{1}-u_{2}\right\|_{W_{0}^{1, p}\left(\Omega, \omega_{1}, \omega_{2}\right)}^{p},
\end{gathered}
$$

where $\gamma=\min \{\lambda, \Lambda\}$.

Therefore, the operator $A$ is strongly monotone, and this implies that $A$ is strictly monotone. Moreover, from Remark 1 and (H7) it follows that

$$
\begin{aligned}
\langle A u, u\rangle= & B(u, u)=B_{1}(u, u)+B_{2}(u, u)=\int_{\Omega} \mathcal{A}(x, \nabla u) \cdot \nabla u \omega_{2} d x+\int_{\Omega} b(x, u) u \omega_{1} d x+ \\
& +\int_{\Omega} g u^{2} d x \geq \int_{\Omega} \lambda|\nabla u|^{p} \omega_{2} d x+\int_{\Omega} \Lambda|u|^{p} \omega_{1} d x \geq \gamma\|u\|_{W_{0}^{1, p}\left(\Omega, \omega_{1}, \omega_{2}\right)}^{p},
\end{aligned}
$$

where $\gamma=\min \{\lambda, \Lambda\}>0$. Since $p>2$, we have

$$
\frac{\langle A u, u\rangle}{\|u\|_{W_{0}^{1, p}\left(\Omega, \omega_{1}, \omega_{2}\right)}} \rightarrow+\infty, \text { as }\|u\|_{W_{0}^{1, p}\left(\Omega, \omega_{1}, \omega_{2}\right)} \rightarrow+\infty,
$$

that is, $A$ is coercive.

Step 5. We need to show that the operator $A$ is continuous. Let $u_{m} \rightarrow u$ in $W_{0}^{1, p}\left(\Omega, \omega_{1}, \omega_{2}\right)$ as $m \rightarrow \infty$. Then

$$
\begin{gathered}
\left|B_{2}\left(u_{m}, \varphi\right)-B_{2}(u, \varphi)\right| \leq \sum_{j=1}^{n} \int_{\Omega}\left|\mathcal{A}_{j}\left(x, \nabla u_{m}\right)-\mathcal{A}_{j}(x, \nabla u) \| D_{j} \varphi\right| \omega_{2} d x= \\
=\sum_{j=1}^{n} \int_{\Omega}\left|F_{j} u_{m}-F_{j} u\right|\left|D_{j} \varphi\right| \omega_{2} d x \leq \sum_{j=1}^{n}\left\|F_{j} u_{m}-F_{j} u\right\|_{L^{p^{\prime}\left(\Omega, \omega_{2}\right)}}\left\|D_{j} \varphi\right\|_{L^{p}\left(\Omega, \omega_{2}\right)} \leq \\
\leq \sum_{j=1}^{n}\left\|F_{j} u_{m}-F_{j} u\right\|_{L^{p^{\prime}\left(\Omega, \omega_{2}\right)}}\|\varphi\|_{W_{0}^{1, p}\left(\Omega, \omega_{1}, \omega_{2}\right)}, \\
\left|B_{1}\left(u_{m}, \varphi\right)-B_{1}(u, \varphi)\right|=\left|\int_{\Omega}\left(b\left(x, u_{m}\right)-b(x, u)\right) \varphi \omega_{1} d x+\int_{\Omega} g \varphi\left(u_{m}-u\right) d x\right| \leq \\
\leq \int_{\Omega}\left|G u_{m}-G u\left\|\varphi\left|\omega_{1} d x+\int_{\Omega}\right| g\right\| \varphi\left\|u_{m}-u \mid d x \leq\right\| G u_{m}-G u\left\|_{L^{p^{\prime}}\left(\Omega, \omega_{1}\right)}\right\| \varphi \|_{L^{p}\left(\Omega, \omega_{1}\right)}+\right.
\end{gathered}
$$




$$
\begin{gathered}
+\left\|g / \omega_{1}\right\|_{L^{q}\left(\Omega, \omega_{1}\right)}\|\varphi\|_{L^{p}\left(\Omega, \omega_{1}\right)}\left\|u_{m}-u\right\|_{L^{p}\left(\Omega, \omega_{1}\right)} \leq\left\|G u_{m}-G u\right\|_{L^{p^{\prime}\left(\Omega, \omega_{1}\right)}}\|\varphi\|_{W_{0}^{1, p}\left(\Omega, \omega_{1}, \omega_{2}\right)}+ \\
+\left\|g / \omega_{1}\right\|_{L^{q}\left(\Omega, \omega_{1}\right)}\|\varphi\|_{W_{0}^{1, p}\left(\Omega, \omega_{1}, \omega_{2}\right)}\left\|u_{m}-u\right\|_{W_{0}^{1, p}\left(\Omega, \omega_{1}, \omega_{2}\right)},
\end{gathered}
$$

for all $\varphi \in W_{0}^{1, p}\left(\Omega, \omega_{1}, \omega_{2}\right)$. Hence,

$$
\begin{aligned}
\mid B\left(u_{m}, \varphi\right) & -B(u, \varphi)|\leq| B_{1}\left(u_{m}, \varphi\right)-B_{1}(u, \varphi)|+| B_{2}\left(u_{m}, \varphi\right)-B_{2}(u, \varphi) \mid \leq \\
\leq & {\left[\sum_{j=1}^{n}\left\|F_{j} u_{m}-F_{j} u\right\|_{L^{p^{\prime}\left(\Omega, \omega_{2}\right)}}+\left\|G u_{m}-G u\right\|_{L^{p^{\prime}\left(\Omega, \omega_{1}\right)}}+\right.} \\
& \left.+\left\|g / \omega_{1}\right\|_{L^{q}\left(\Omega, \omega_{1}\right)}\left\|u_{m}-u\right\|_{W_{0}^{1, p}\left(\Omega, \omega_{1}, \omega_{2}\right)}\right]\|\varphi\|_{W_{0}^{1, p}\left(\Omega, \omega_{1}, \omega_{2}\right)} .
\end{aligned}
$$

Then we obtain

$$
\begin{gathered}
\left\|A u_{m}-A u\right\|_{*} \leq \sum_{j=1}^{n}\left\|F_{j} u_{m}-F_{j} u\right\|_{L^{p^{\prime}\left(\Omega, \omega_{2}\right)}}+ \\
+\left\|G u_{m}-G u\right\|_{L^{p^{\prime}\left(\Omega, \omega_{1}\right)}}+\left\|g / \omega_{1}\right\|_{L^{q}\left(\Omega, \omega_{1}\right)}\left\|u_{m}-u\right\|_{W_{0}^{1, p}\left(\Omega, \omega_{1}, \omega_{2}\right)} .
\end{gathered}
$$

Therefore, $\left\|A u_{m}-A u\right\|_{*} \rightarrow 0$ as $m \rightarrow+\infty$ by (3) and (4). Hence, $A$ is continuous and this implies that $A$ is hemicontinuous.

By Theorem 3, the operator equation $A u=T$ has a unique solution $u \in W_{0}^{1, p}\left(\Omega, \omega_{1}, \omega_{2}\right)$ and it is the unique solution for problem $(\mathrm{P})$.

Step 6. According to Definition 1, we have

$$
B(u, u)=B_{1}(u, u)+B_{2}(u, u)=T(u) .
$$

Therefore, using Remark 1, (H7), we obtain

$$
\begin{gathered}
B_{1}(u, u)+B_{2}(u, u)=\int_{\Omega} \mathcal{A}(x, \nabla u) \cdot \nabla u \omega_{2} d x+\int_{\Omega} b(x, u) u \omega_{1} d x+\int_{\Omega} g u^{2} d x \geq \\
\geq \int_{\Omega} \lambda|\nabla u|^{p} \omega_{2} d x+\int_{\Omega} \Lambda|u|^{p} \omega_{1} d x \geq \gamma\|u\|_{W_{0}^{1, p}\left(\Omega, \omega_{1}, \omega_{2}\right)}^{p},
\end{gathered}
$$

where $\gamma=\min \{\lambda, \Lambda\}>0$, and by (H9) we have

$$
\begin{gathered}
T(u)=\int_{\Omega} f_{0} u d x+\sum_{j=1}^{n} \int_{\Omega} f_{j} D_{j} u d x \leq\left\|f_{0} / \omega_{1}\right\|_{L^{p^{\prime}}\left(\Omega, \omega_{1}\right)}\|u\|_{L^{p}\left(\Omega, \omega_{1}\right)}+ \\
+\sum_{j=1}^{n}\left\|f_{j} / \omega_{2}\right\|_{L^{p^{\prime}(\Omega)}}\left\|D_{j} u\right\|_{L^{p}\left(\Omega, \omega_{2}\right)} \leq\left(\left\|f_{0} / \omega_{1}\right\|_{L^{p^{\prime}\left(\Omega, \omega_{1}\right)}}+\sum_{j=1}^{n}\left\|f_{j} / \omega_{2}\right\|_{L^{p^{\prime}\left(\Omega, \omega_{2}\right)}}\right)\|u\|_{W_{0}^{1, p}\left(\Omega, \omega_{1}, \omega_{2}\right)} .
\end{gathered}
$$

Therefore, in (6), we obtain

$$
\gamma\|u\|_{W_{0}^{1, p}\left(\Omega, \omega_{1}, \omega_{2}\right)}^{p} \leq\left(\left\|f_{0} / \omega_{1}\right\|_{L^{p^{\prime}\left(\Omega, \omega_{1}\right)}}+\sum_{j=1}^{n}\left\|f_{j} / \omega_{2}\right\|_{L^{p^{\prime}\left(\Omega, \omega_{2}\right)}}\right)\|u\|_{W_{0}^{1, p}\left(\Omega, \omega_{1}, \omega_{2}\right)} .
$$


Since $p>2$, we have

$$
\|u\|_{W_{0}^{1, p}\left(\Omega, \omega_{1}, \omega_{2}\right)} \leq \frac{1}{\gamma^{p^{\prime} / p}}\left(\left\|\frac{f_{0}}{\omega_{1}}\right\|_{L^{p^{\prime}\left(\Omega, \omega_{1}\right)}}+\sum_{j=1}^{n}\left\|\frac{f_{j}}{\omega_{2}}\right\|_{L^{p^{\prime}\left(\Omega, \omega_{2}\right)}}\right)^{p^{\prime} / p} .
$$

Proof of Corollary 1. If $u_{1}, u_{2} \in W_{0}^{1, p}\left(\Omega, \omega_{1}, \omega_{2}\right)$ are solutions of $\left(P_{1}\right)$ and $\left(P_{2}\right)$ respectively, then

$$
\begin{aligned}
& \int_{\Omega} \mathcal{A}\left(x, \nabla u_{1}\right) \cdot \nabla \varphi \omega_{2} d x+\int_{\Omega} b\left(x, u_{1}\right) \varphi \omega_{1} d x+\int_{\Omega} g u_{1} \varphi d x=\int_{\Omega} f_{0} \varphi d x+\sum_{j=1}^{n} \int_{\Omega} f_{j} D_{j} \varphi d x \\
& \int_{\Omega} \mathcal{A}\left(x, \nabla u_{2}\right) \cdot \nabla \varphi \omega_{2} d x+\int_{\Omega} b\left(x, u_{2}\right) \varphi \omega_{1} d x+\int_{\Omega} g u_{2} \varphi d x=\int_{\Omega} \tilde{f}_{0} \varphi d x+\sum_{j=1}^{n} \int_{\Omega} \tilde{f}_{j} D_{j} \varphi d x
\end{aligned}
$$

for all $\varphi \in W_{0}^{1, p}\left(\Omega, \omega_{1}, \omega_{2}\right)$. In particular, we have

$$
\begin{gathered}
\int_{\Omega}\left(\mathcal{A}\left(x, \nabla u_{1}\right)-\mathcal{A}\left(x, \nabla u_{2}\right)\right) \cdot \nabla\left(u_{1}-u_{2}\right) \omega_{2} d x+\int_{\Omega}\left(b\left(x, u_{1}\right)-b\left(x, u_{2}\right)\right)\left(u_{1}-u_{2}\right) \omega_{1} d x+ \\
\quad+\int_{\Omega} g\left(u_{1}-u_{2}\right)^{2} d x=\int_{\Omega}\left(f_{0}-\tilde{f}_{0}\right)\left(u_{1}-u_{2}\right) d x+\sum_{j=1}^{n} \int_{\Omega}\left(f_{j}-\tilde{f}_{j}\right) D_{j}\left(u_{1}-u_{2}\right) d x
\end{gathered}
$$

for $\varphi=u_{1}-u_{2}$.

(i) By (H2), we have

$$
\int_{\Omega}\left(\mathcal{A}\left(x, \nabla u_{1}\right)-\mathcal{A}\left(x, \nabla u_{2}\right)\right) \cdot \nabla\left(u_{1}-u_{2}\right) \omega_{2} d x \geq \lambda \int_{\Omega}\left|\nabla\left(u_{1}-u_{2}\right)\right|^{p} \omega_{2} d x,
$$

and by (H5), we obtain $\int_{\Omega}\left(b\left(x, u_{1}\right)-b\left(x, u_{2}\right)\right)\left(u_{1}-u_{2}\right) \omega_{1} \geq \Lambda \int_{\Omega}\left|u_{1}-u_{2}\right|^{p} \omega_{1} d x$.

(ii) By (H7) we have $\int_{\Omega} g\left(u_{1}-u_{2}\right)^{2} d x \geq 0$.

Hence, in (7), if $\gamma=\min \{\lambda, \Lambda\}$, we obtain

$$
\begin{gathered}
\gamma\left\|u_{1}-u_{2}\right\|_{W_{0}^{1, p}\left(\Omega, \omega_{1}, \omega_{2}\right)}^{p} \leq \\
\leq\left(\left\|\left(f_{0}-\tilde{f}_{0}\right) / \omega_{1}\right\|_{L^{p^{\prime}}\left(\Omega, \omega_{1}\right)}+\sum_{j=1}^{n}\left\|\left(f_{j}-\tilde{f}_{j}\right) / \omega_{2}\right\|_{L^{p^{\prime}\left(\Omega, \omega_{2}\right)}}\right)\left\|u_{1}-u_{2}\right\|_{W_{0}^{1, p}\left(\Omega, \omega_{1}, \omega_{2}\right)} .
\end{gathered}
$$

Therefore,

$$
\left\|u_{1}-u_{2}\right\|_{W_{0}^{1, p}\left(\Omega, \omega_{1}, \omega_{2}\right)} \leq \frac{1}{\gamma^{p / p^{\prime}}}\left(\left\|\left(f_{0}-\tilde{f}_{0}\right) / \omega_{1}\right\|_{L^{p^{\prime}\left(\Omega, \omega_{1}\right)}}+\sum_{j=1}^{n}\left\|\left(f_{j}-\tilde{f}_{j}\right) / \omega_{2}\right\|_{L^{p^{\prime}\left(\Omega, \omega_{2}\right)}}\right)^{p / p^{\prime}} .
$$

Proof of Corollary 2. By Corollary 1 if $u_{m}$ and $u$ are solutions of $\left(P_{m}\right)$ and $(P)$ respectively, then

$$
\left\|u_{m}-u\right\|_{W_{0}^{1, p}\left(\Omega, \omega_{1}, \omega_{2}\right)} \leq \frac{1}{\gamma^{p / p^{\prime}}}\left(\left\|\left(f_{0 m}-f_{0}\right) / \omega_{1}\right\|_{L^{p^{\prime}}\left(\Omega, \omega_{1}\right)}+\sum_{j=1}^{n}\left\|\left(f_{j m}-f_{j}\right) / \omega_{2}\right\|_{L^{p^{\prime}\left(\Omega, \omega_{2}\right)}}\right)^{p / p^{\prime}} .
$$

Since $\frac{f_{0 m}}{\omega_{1}} \rightarrow \frac{f_{0}}{\omega_{1}}$ in $L^{p^{\prime}}\left(\Omega, \omega_{1}\right), \frac{f_{j m}}{\omega_{2}} \rightarrow \frac{f_{j}}{\omega_{2}}$ in $L^{p^{\prime}}\left(\Omega, \omega_{2}\right)(j=1, \ldots, n)$, we obtain the convergence $u_{m} \rightarrow u$ in $W_{0}^{1, p}\left(\Omega, \omega_{1}, \omega_{2}\right)$. 
Example 1. Let $\Omega=\left\{(x, y) \in \mathbb{R}^{2}: x^{2}+y^{2}<1\right\}$. We consider the weights functions $\omega_{1}(x, y)=\left(x^{2}+y^{2}\right)^{-1 / 2}$ and $\omega_{2}(x, y)=\left(x^{2}+y^{2}\right)^{-2 / 3}\left(\omega_{1}\right.$ and $\omega_{2}$ are $A_{3}$-weights, $\left.p=3\right)$, and the functions

$$
\begin{gathered}
\mathcal{A}: \Omega \times \mathbb{R}^{2} \rightarrow \mathbb{R}^{2}, \mathcal{A}((x, y), \xi)=h_{2}(x, y)|\xi| \xi \\
b: \Omega \times \mathbb{R} \rightarrow \mathbb{R}, b((x, y), \eta)=\eta|\eta|\left(\cos ^{2}(x y)+1\right), \quad g: \Omega \rightarrow \mathbb{R}, g(x, y)=\frac{\sin ^{2}(x y)}{\left(x^{2}+y^{2}\right)^{1 / 12}}
\end{gathered}
$$

with $\lambda=1 / 2, \Lambda=1 / 4, h_{1}(x, y)=\cos ^{2}(x y)+1, h_{2}(x, y)=2 \mathrm{e}^{x^{2}+y^{2}}$ and $q=3$. Let us consider the partial differential operator

$$
L u(x, y)=-\operatorname{div}\left[\mathcal{A}((x, y), \nabla u) \omega_{2}(x, y)\right]+b((x, y), u) \omega_{1}(x, y)+g(x, y) u .
$$

By Theorem 1, the problem

$$
(P) \begin{cases}L u(x, y)=\frac{\cos (x y)}{\left(x^{2}+y^{2}\right)}-\frac{\partial}{\partial x}\left(\frac{\sin (x y)}{\left(x^{2}+y^{2}\right)^{2}}\right)-\frac{\partial}{\partial y}\left(\frac{\cos (x y)}{\left(x^{2}+y^{2}\right)^{2}}\right) & \text { in } \Omega, \\ u(x, y)=0 & \text { on } \partial \Omega,\end{cases}
$$

has a unique solution $u \in W_{0}^{1,3}\left(\Omega, \omega_{1}, \omega_{2}\right)$.

Remark. To verify the conditions (H2) and (H5) we use the inequality

$$
\left(|\xi|^{p-2} \xi-|\tilde{\xi}|^{p-2} \tilde{\xi}\right) \cdot(\xi-\tilde{\xi}) \geq \frac{1}{2^{p-1}}(|\xi|+|\tilde{\xi}|)^{p-2}|\xi-\tilde{\xi}|^{2}
$$

for every $\xi, \tilde{\xi} \in \mathbb{R}^{n}$ and $1<p<\infty$ (see Proposition 17.3 in [1]). For $p=3$, we have

$$
(|\xi| \xi-|\tilde{\xi}| \tilde{\xi}) \cdot(\xi-\tilde{\xi}) \geq \frac{1}{4}(|\xi|+|\tilde{\xi}|)|\xi-\tilde{\xi}|^{2}
$$

and since $|\xi|+|\tilde{\xi}| \geq|\xi-\tilde{\xi}|$, we obtain $(|\xi| \xi-|\tilde{\xi}| \tilde{\xi}) \cdot(\xi-\tilde{\xi}) \geq \frac{1}{4}|\xi-\tilde{\xi}|^{3}$. Hence, using that $h_{2}(x, y)=2 \mathrm{e}^{x^{2}+y^{2}} \geq 2$ in $\Omega$, we have $(\mathcal{A}((x, y), \xi)-\mathcal{A}((x, y), \tilde{\xi})) \cdot(\xi-\tilde{\xi}) \geq \frac{1}{2}|\xi-\tilde{\xi}|^{3}$. Analogously, we have

$$
(b((x, y), \eta)-b((x, y), \tilde{\eta}))(\eta-\tilde{\eta})=\left(\cos ^{2}(x y)+1\right)(|\eta| \eta-|\tilde{\eta}| \tilde{\eta})(\eta-\tilde{\eta}) \geq \frac{1}{4}|\eta-\tilde{\eta}|^{3} .
$$

Acknowledgements. The author thanks the referee for his/her helpful suggestions which improved the original results.

\section{REFERENCES}

1. M. Chipot, Elliptic equations: an introductory course, Birkhäuser, Berlin, 2009.

2. P. Drábek, J. Milota, Methods of nonlinear analysis, Birkhäuser, Berlin, 2007.

3. E. Fabes, D. Jerison, C. Kenig, The Wiener test for degenerate elliptic equations, Ann. Inst. Fourier, 32 (1982), 151-182. 
4. S. Fučik, O. John and A. Kufner, Function spaces, Noordhoff International Publ., Leyden, 1977.

5. E. Fabes, C. Kenig, R. Serapioni, The local regularity of solutions of degenerate elliptic equations, Comm. Partial Differenctial Equations, 7 (1982), 77-116.

6. B. Franchi, R. Serapioni, Pointwise estimates for a class of strongly degenerate elliptic operators: a geometrical approch, Ann. Sc. Norm. Super. Pisa Cl. Sci.(5), 14 (1987), 527-568.

7. J. Garcia-Cuerva, J.L. Rubio de Francia, Weighted norm inequalities and related topics, North-Holland Mathematics Studies, 116, (1985).

8. J. Heinonen, T. Kilpeläinen, O. Martio, Nonlinear Potential Theory of Degenerate Elliptic Equations, Oxford Math. Monographs, Clarendon Press, 1993.

9. A. Kufner, Weighted Sobolev spaces, John Wiley \& Sons, Chichester, 1985.

10. A. Kufner, B.Opic, How to define reasonably weighted Sobolev spaces, Comment. Math. Univ. Carolin., 25 (1984), 573-554.

11. B. Muckenhoupt, Weighted norm inequalities for the Hardy maximal function, Trans. Amer. Math. Soc., 165 (1972), 207-226.

12. A. Torchinsky, Real-variable methods in harmonic analysis, Academic Press, San Diego, 1986.

13. B.O. Turesson, Nonlinear potential theory and weighted Sobolev spaces, Lecture Notes in Math., V.1736, Springer-Verlag, 2000.

14. E. Zeidler, Nonlinear functional analysis and its applications, vol.II/A, Springer-Verlag, New York, 1990.

15. E. Zeidler, Nonlinear Functional Analysis and its Applications, V.II/B, Springer-Verlag, New Yor,k 1990.

Department of Mathematics

State University of Londrina, Brazil

accava@gmail.com 